\title{
THE INCIDENCE OF ADOLESCENT PREGNANCY AT CLINIC OF GYNECOLOGY AND OBSTETRICS OF CLINICAL CENTER KRAGUJEVAC
}

\author{
Marija Bicanin Ilic ${ }^{1}$ and Aleksandra Dimitrijevic ${ }^{1}$ \\ ${ }^{I}$ Clinic of Gynecology and Obstetrics, Clinical Centre Kragujevac, Kragujevac, Serbia
}

Received: 26.04 .2019 . Accepted: 03.05.2019.

\section{Corresponding author:}

\section{Marija Bicanin Ilic, MD}

Clinic of Gynecology and Obstetrics, Clinical Centre Kragujevac

30 Zmaj Jovina Street, Kragujevac, Serbia

Phone: +381642210218

E-mail: abahelica@hotmail.com

\section{sciendo}

UDK: 618.2-053.6(497.11)"2007/2019"

Ser J Exp Clin Res 2022; 23(1): 59-66

DOI: $10.2478 /$ sjecr-2019-0028

\begin{abstract}
Adolescent pregnancy belongs to a group of high-risk pregnancies with high maternal and fetal mortality and morbidity rate with high prevalence globally (11\%). The aim of this observational study is to show the incidence of adolescent deliveries in relation to the total number of births in the twelve-year period from 2007 to 2019 at the Department of Gynecology and Obstetrics of Clinical Center in Kragujevac from medical protocols and patients' medical records. By evaluating the data of our research, we noticed a continuing decrease in the percentage of adolescents that give births each year. The total number of births in our clinic in this twelve-year period was 26544, and the number of teenage deliveries was 390 (1.74\%), which is in accordance with the results of a similar research which was conducted in our clinic in the period from 2002 to 2007 (16.1\%). However, despite the increase in the number of caesarean sections, Apgar score of newborn babies was similar to the results of previous tests - 8.31 which proves that the increase in caesarean sections is not correlated with growth of Apgar score. The average pregnancy duration of adolescents is similar as in the previous five-year period (37.5 w.g.), while in the general population of pregnant women it is 39.2 w.g which represents a statistically significant difference. The main causes of poor outcomes of adolescent pregnancies are biological immaturity of mothers, poor health care, that comes from poor socio-demographic conditions, as well as emotional stress. It is necessary to change the attitude of society towards these young people, give them support in the environment they live and provide them with better health care and social treatment.
\end{abstract}

Keywords: Adolescent pregnancy, teenage pregnancy, young age childbirth. 


\section{INTRODUCTION}

According to the World Health Organization (WHO) definition, adolescence presents forming full sexual maturity, maturing of person from the psyche of the child to the psyche of an adult and transition from full social and economic dependence to the relative independence (1). This life period of a woman is followed by turbulent psychosocial events and often complicated by dyscoordination of mental and physical maturation. Modern hygiene and dietary conditions have led to the occurrence of girl's menarche earlier, that together, followed by the customs and attitudes of the surroundings, results in sexual relations at the early age. Adolescents are usually not aware of the risks and dangers of unprotected sexual behavior and they are not mentally mature to face the consequences of the act. Adolescent pregnancy is usually the product of the mentioned factors. It belongs to a group of highrisk pregnancies with high maternal and fetal mortality and morbidity rate. Pregnancy at this age has been reported as the leading cause of death in adolescent girls in low- and middleincome countries (2).

Insufficient biological maturity of pregnant girls, poor socio epidemiological living conditions and inadequate medical care are key factors of pregnancy complications amongwhich the most common are: premature birth, intrauterine growth restriction, pregnancy induced hypertension $(\mathrm{PIH})$, anemia, infections and fetal anomaly (1). Partial progesterone resistance that persists into adolescent years may compromise the physiological transformation of the spiral arteries and predispose for defective placentation in the case of pregnancy. The major obstetric syndromes caused by impaired placental bed spiral artery remodeling are prevalent in teenage pregnancies, including preeclampsia, fetal growth restriction, and spontaneous preterm labor (3). Some studies report that the adverse outcomes are due to physiological and anatomical factors associated with young maternal age, while others report that they are due to external factors such as socioeconomic status, social support, inadequate antenatal care and other behavioural determinants associated with adolescence (4). Almost all assessments of adolescent pregnancies are based on chronological age. Gynecologic age is defined as age in years at conception minus age at menarche and it is an indicator of physiological maturity. Low gynecologic age is associated with an increased rate of obstetric and perinatal complications in adolescent pregnancies. Although the main aim is the prevention of adolescent pregnancies, a detailed evaluation of such pregnancies including determination of the gynecological age together with a multidisciplinary approach may decrease potential complications (5).

According to WHO statistics, about 16 million girls aged 15 to 19 give birth each year, mostly in low- and middle- income countries, and about 3 million have an abortion. Complications during pregnancy and childbirth are the second cause of death of girls in this age group. Young mothers, and particularly teenage mothers, are a vulnerable group at high risk of poor mental health outcomes compared to mothers aged 25 and above (6).

About $11 \%$ of all pregnancies globally belong to adolescent pregnancies (7). Since 1990 there has been a downward trend in adolescent pregnancies which is associated with more massive educating youth about the risks and consequences of unprotected sexual relation and massive use of condoms.

The aim of this study is to determine the incidence of adolescent deliveries in relation to the total number of births in the twelve-year period from 2007 to 2019 at the Department of Gynecology and Obstetrics of Clinical Center in Kragujevac by collecting the data about the maternal age, body weight and Apgar score of newborns, indications for instrumental delivery and frequency of caesarean section.

\section{METHODOLOGY}

\section{Ethical concerns}

This observational study was performed in accordance with all relevant ethical principles.

\section{Design of the study}

This study was designed as retrospective cross-sectional observational clinical study which evaluated the incidence of adolescent pregnancy during twelve-year period from 2007 to 2019 at the Department of Gynecology and Obstetrics of Clinical Center in Kragujevac from medical protocols and patients' medical records.

\section{Population of the study}

The study included all medical data regarding adolescent pregnancy from birth protocols and caesarean sections protocols and patients' medical records during past twelve years. The main inclusion criteria were adolescent age (12-18 years old) and confirmed pregnancy.

\section{Data processing and analysis}

All data were processed in Excel, presented in tables and charts, statistically expressed by mean and by percentile. 


\section{RESULTS}

\section{Prevalence of adolescent births}

During past twelve years, the highest number of adolescent births occurred in 2009, then in 2007 and 2008 in eighteen years old mothers. On the other hand, the smallest count of adolescent pregnancies was in last two years (2017 and 2018 ) in the youngest mothers (Table 1).
In percent, from all pregnancies over past twelve years, the highest incidence was in 2009, then in 2007 and 2008. On the other hand, 2017 and 2018 were years with the lowest incidence (Figure 1).

Comparative presentation of the total number of births and births by adolescents by age shows that the first four years have the highest frequency compared with other years and that the dynamics of frequency was different over the twelve years study period (Table 2).

Table 1. Total number of adolescent deliveries classified by age of mothers

\begin{tabular}{|c|c|c|c|c|c|c|c|c|c|c|c|c|}
\cline { 2 - 14 } \multicolumn{1}{c|}{} & $\mathbf{2 0 0 7}$ & $\mathbf{2 0 0 8}$ & $\mathbf{2 0 0 9}$ & $\mathbf{2 0 1 0}$ & $\mathbf{2 0 1 1}$ & $\mathbf{2 0 1 2}$ & $\mathbf{2 0 1 3}$ & $\mathbf{2 0 1 4}$ & $\mathbf{2 0 1 5}$ & $\mathbf{2 0 1 6}$ & $\mathbf{2 0 1 7}$ & $\mathbf{2 0 1 8}$ \\
\hline $\mathbf{1 2}$ & 1 & 0 & 0 & 0 & 0 & 0 & 0 & 0 & 0 & 0 & 0 & 0 \\
\hline $\mathbf{1 3}$ & 0 & 0 & 0 & 0 & 0 & 0 & 0 & 0 & 0 & 0 & 0 & 0 \\
\hline $\mathbf{1 4}$ & 1 & 0 & 0 & 1 & 2 & 0 & 0 & 0 & 0 & 0 & 0 & 0 \\
\hline $\mathbf{1 5}$ & 0 & 2 & 4 & 1 & 1 & 3 & 3 & 2 & 0 & 0 & 1 & 0 \\
\hline $\mathbf{1 6}$ & 4 & 9 & 6 & 9 & 4 & 2 & 3 & 4 & 4 & 0 & 1 & 2 \\
\hline $\mathbf{1 7}$ & 18 & 14 & 15 & 11 & 9 & 7 & 9 & 8 & 5 & 10 & 4 & 1 \\
\hline $\mathbf{1 8}$ & 28 & 26 & 30 & 21 & 17 & 22 & 16 & 11 & 10 & 15 & 5 & 8 \\
\hline$\Sigma$ & $\mathbf{5 2}$ & $\mathbf{5 1}$ & $\mathbf{5 5}$ & $\mathbf{4 3}$ & $\mathbf{3 3}$ & $\mathbf{3 4}$ & $\mathbf{3 1}$ & $\mathbf{2 5}$ & $\mathbf{1 9}$ & $\mathbf{2 5}$ & $\mathbf{1 1}$ & $\mathbf{1 1}$ \\
\hline
\end{tabular}

Figure 1. Frequency of adolescent deliveries

$003 \%$

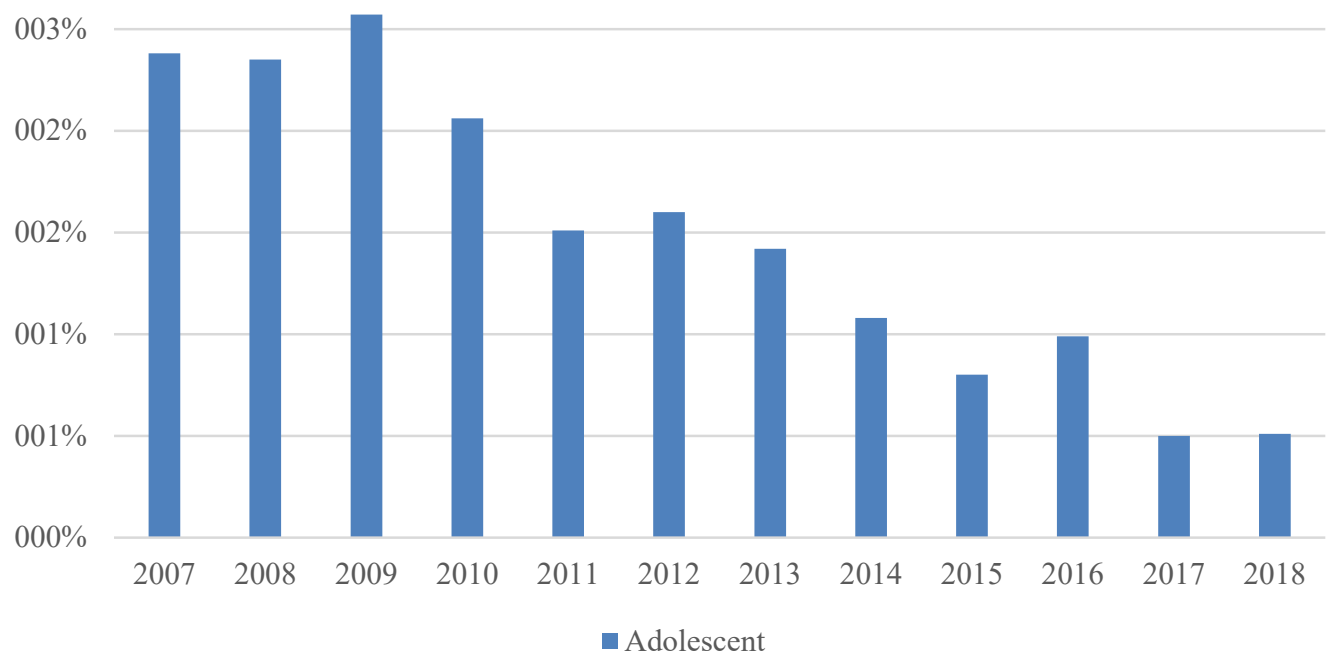


Table 2. Comparative presentation of the total number of births and deliveries by adolescents from 2007 to 2018

\begin{tabular}{|l|c|c|c|c|c|c|c|c|c|c|c|c|}
\cline { 2 - 26 } \multicolumn{1}{c|}{} & $\mathbf{2 0 0 7}$ & $\mathbf{2 0 0 8}$ & $\mathbf{2 0 0 9}$ & $\mathbf{2 0 1 0}$ & $\mathbf{2 0 1 1}$ & $\mathbf{2 0 1 2}$ & $\mathbf{2 0 1 3}$ & $\mathbf{2 0 1 4}$ & $\mathbf{2 0 1 5}$ & $\mathbf{2 0 1 6}$ & $\mathbf{2 0 1 7}$ & $\mathbf{2 0 1 8}$ \\
\hline Total number of births & 2180 & 2171 & 2138 & 2088 & 2185 & 2121 & 2181 & 2302 & 2352 & 2511 & 2170 & 2151 \\
\hline adolescents & 52 & 51 & 55 & 43 & 33 & 34 & 31 & 25 & 19 & 25 & 11 & 11 \\
\hline percentage & 2.38 & 2.34 & 2.57 & 2.06 & 1.51 & 1.60 & 1.42 & 1.08 & 0.80 & 0.99 & 0.50 & 0.51 \\
\hline
\end{tabular}

\section{Apgar score of newborns from adolescent pregnancies}

Table 3 shows distribution of Apgar scores of newborns in adolescent pregnancies during twelve-year period. Most newborns were born with 8-10 Apgar score (91.3\%) (Table 3).

Table 3. Apgar score of newborns from adolescent pregnancies

\begin{tabular}{|c|c|c|c|c|c|c|c|c|c|c|c|c|}
\hline & $\mathbf{2 0 0 7}$ & $\mathbf{2 0 0 8}$ & $\mathbf{2 0 0 9}$ & $\mathbf{2 0 1 0}$ & $\mathbf{2 0 1 1}$ & $\mathbf{2 0 1 2}$ & $\mathbf{2 0 1 3}$ & $\mathbf{2 0 1 4}$ & $\mathbf{2 0 1 5}$ & $\mathbf{2 0 1 6}$ & $\mathbf{2 0 1 7}$ & $\mathbf{2 0 1 8}$ \\
\hline $\begin{array}{c}\mathbf{5} \mathbf{7} \\
\mathbf{8 . 9 7 \% )}\end{array}$ & 8 & 8 & 4 & 2 & 1 & 3 & 1 & 2 & 1 & 1 & 3 & 1 \\
\hline $\begin{array}{c}\mathbf{8 - 1 0} \\
(\mathbf{9 1 . 3 \% )}\end{array}$ & 44 & 43 & 51 & 41 & 32 & 31 & 30 & 23 & 18 & 24 & 8 & 10 \\
\hline Total number & 52 & 51 & 55 & 43 & 33 & 34 & 31 & 25 & 19 & 25 & 11 & 11 \\
\hline
\end{tabular}

\section{Gestational age of newborns from adolescent pregnancies at the moment of childbirth}

Table 4 shows distribution of gestational age of adolescent pregnancies at the moment of childbirth. The highest frequency of adolescent pregnancies resulted in birth at $32-42$ g.w. (62.82\%), then $37-38$ g.w. (21.28\%) and $\leq 36.6$ g.w. (15.64\%) (Table 4).

Table 4. Gestational age of adolescent pregnancies at the moment of childbirth

\begin{tabular}{|c|c|c|c|c|c|c|c|c|c|c|c|c|c|}
\hline & 2007 & 2008 & 2009 & 2010 & 2011 & 2012 & 2013 & 2014 & 2015 & 2016 & 2017 & 2018 & $\begin{array}{c}\text { Total } \\
\%\end{array}$ \\
\hline$\leq 36.6$ & 9 & 5 & 8 & 6 & 3 & 6 & 1 & 3 & 4 & 8 & 4 & 4 & $(15.64 \%)$ \\
\hline 37-38 g.w. & 5 & 9 & 9 & 7 & 5 & 5 & 12 & 7 & 3 & 12 & 4 & 5 & $(21.28 \%)$ \\
\hline 39-42 g.w. & 38 & 37 & 38 & 30 & 25 & 23 & 17 & 15 & 12 & 5 & 3 & 2 & $(62.82 \%)$ \\
\hline
\end{tabular}

Ratio of average body weight of newborns from adolescent pregnancies with body weights of newborns according to anthropometric standards

Figure 2 shows ratio of the average body weight of newborns from adolescent pregnancies with body weights of newborns according to anthropometric standards. The averages of body mass of newborns are higher in higher g.w. (Figure 2). 
Figure 2. Ratio of average body weight of newborns from adolescent pregnancies with body weights of newborns according to anthropometric standards. (11)

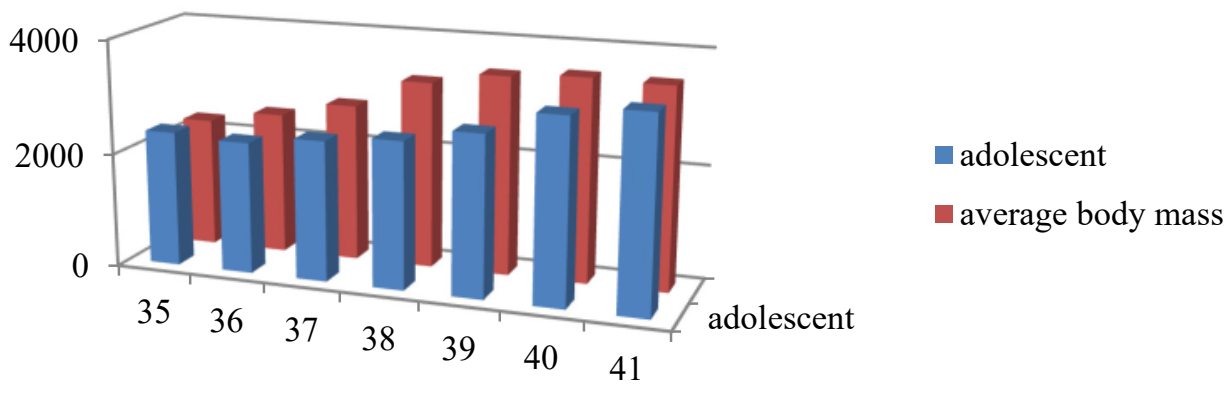

\section{Distribution of caesarean section in adolescents compared to general population of women}

In Table 5 and Figure 3 are presented distribution of caesarean section in adolescents compared to general population of women. The highest ratio was observed in 2015, then in 2009, and the lowest ratio in 2011 (Table 5).

Table 5. The ratio of births by caesarean section in adolescents and in the general population of women.

\begin{tabular}{|c|c|c|c|c|c|c|}
\cline { 2 - 7 } \multicolumn{1}{c|}{} & 2007 & 2008 & 2009 & 2010 & 2011 & 2012 \\
\hline $\begin{array}{c}\text { Adolescent } \\
\text { C-sections (Total } \\
8.97 \%)\end{array}$ & 52 & 51 & 55 & 43 & 33 & 34 \\
\hline $\begin{array}{c}4 \\
(7.69 \%)\end{array}$ & $\begin{array}{c}3 \\
(5.88 \%)\end{array}$ & $\begin{array}{c}6 \\
(10.99 \%)\end{array}$ & $\begin{array}{c}1 \\
(2.35 \%)\end{array}$ & $\begin{array}{c}0 \\
(0 \%)\end{array}$ & $\begin{array}{c}4 \\
(11.74 \%)\end{array}$ \\
\hline $\begin{array}{c}\text { Adolescent } \\
\text { A- sections (Total } \\
8.97 \%)\end{array}$ & 31 & 2014 & 2015 & 2016 & 2017 & 2018 \\
\hline
\end{tabular}

Figure 3. The ratio of births by caesarean section in adolescents and in the general population of women.

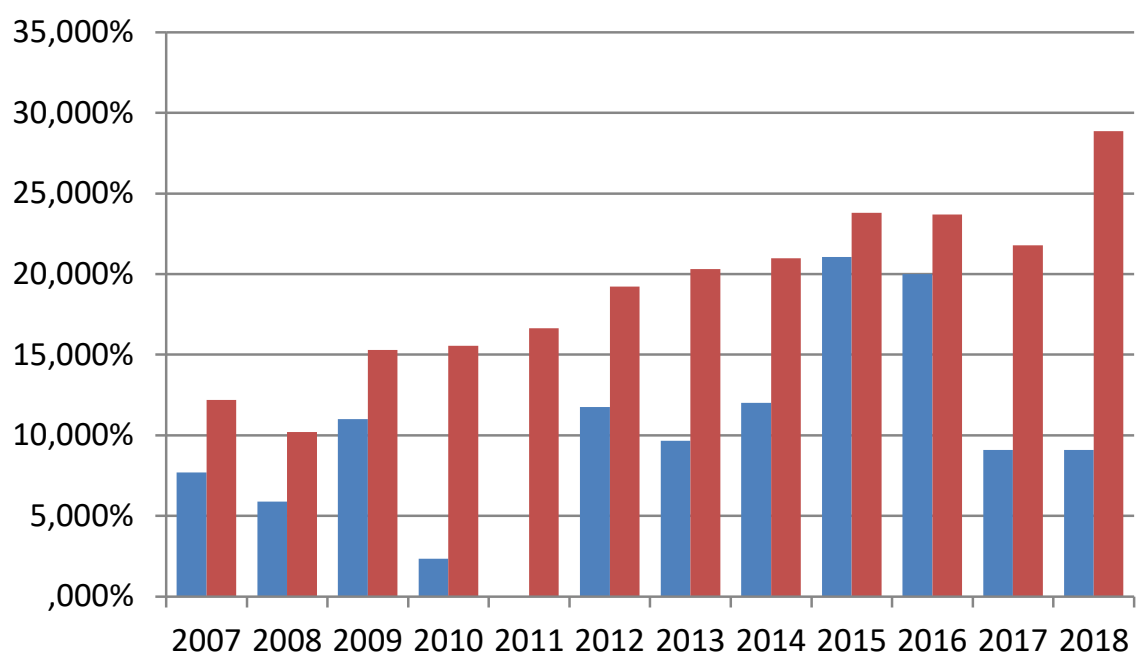




\section{DISCUSSION}

By evaluating the data of our research, we noticed a continuing decrease in the percentage of adolescents that give births each year (Fig. 1).

The total number of births in our clinic in this twelve-year period was 26544, and the number of teenage births was 390 $(1.46 \%)$, which is in accordance with results of a similar research which was conducted in our clinic in the period from 2002 to 2007 (16.1\% expressed in percentage) (8). Both studies showed a significant decrease in the percentage of these deliveries compared to the $90^{\prime}$ of the last century when this percentage was reaching $4.13 \%$. It is far below the world level, which reaches $11 \%(9)$.

We would like to claim that the cause of this trend is better health care provided for young people, education about the risks of early entry into sexual relationships and massive use of condoms among young people. However, more likely cause is greater availability of abortions in private practice that young people easily decide to do. Data on the number of pregnancies terminated in this way do not exist, because they do not report abortions despite legal obligations. Young people terminate pregnancy without being aware of the possible complications of the interventions and health consequences. Emotionally immature young people who do not have adequate support from families and environment, seeking a way out of their unenviable situation, decide for available solutions, which is often an abortion.

Percentage of deliveries completed by caesarean section was $8.97 \%$, which is closer to the results of studies from the $90^{\prime}(6.86 \%)$, while in the period from 2002 to 2007 it was $4.51 \%(8)$. This increase in the number of caesarean sections in a group of adolescents follows the increase in the number of operative deliveries in the general population of pregnant women, but remains far below this percentage. The explanation for this increase can be found in more liberal indications for operative delivery. (9) In the general population of pregnant women, there has been an increase of pregnancies from in vitro fertilization in mature generative age of women and the surgical completion of these pregnancies contributes to the constant increase in the number of caesarean sections.

The percentage of caesarean sections in a group of adolescentsis smaller (Fig. 3) because there is a very small number of the leading indication for surgical termination of a pregnancy that apply to the general population of pregnant women, and those are repeated cesarean section and disproportion. In the group of deliveries by adolescents, most often is vaginal delivery with episiotomy.

However, despite the increase in the number of caesarean sections, Apgar score of newborn babies was similar to the results of previous tests which was 8.31 while in the last twelve-year period it was 8.23 , which proves that the increase in caesarean sections is not correlated with growth of Apgar score.
Among the indications for operative completion of labor in the group of adolescents, leading places belong to Preterm Premature rupture of membranes (PPROM), dystocia, PIH, and fetal asphyxia (9).

The average pregnancy duration of adolescents was similar as in the previous five-year period (37.5 w.g.), while in the general population of pregnant women it is 39.2 w.g. which represents a statistically significant difference.

Preterm delivery together with insufficient body weight and death cases in newborns are the main issues in obstetrics. About $40 \%$ of preterm delivery is caused by infections (10). Even $15.64 \%$ of pregnancies ended giving birth before 37 weeks which is significantly higher than the data from the world literature, which is $10 \%$.

Newborns' body weight from adolescents' pregnancies, expressed by weeks of gestation, significantly deviates from the average weight of newborns in the territory of Šumadija district (Fig. 2), and belongs to the 10 percentile of the body mass of newborns in the territory of Sumadija district (11). Low birth weight is a result of intrauterine growth retardation that occurs in pregnancies complicated by hypertension, malnutrition, genetic anomalies of fetus, etc. Poor prenatal health care is directly related to these complications.

The main causes of poor outcomes of adolescent pregnancies are biological immaturity of mothers, poor health care, that comes from poor socio-demographic conditions, as well as emotional stress. The well-recognized factors of socioeconomic disadvantage, disrupted family structure and low educational level and aspiration appear consistently associated with teenage pregnancy (12).

In European Union countries, this percentage has been declining since 2001, although the trend differs between countries and regions. In Eastern Europe, this percentage is the highest and amounts to $4.17 \%$, while in the North, West and South Europe it amounts to $3.07 \%, 1.82 \%$ and $1.76 \%$, respectively. (13). In the USA, rates for other vulnerable groups, such as runaway and homeless adolescents, adolescents in foster care, and adolescents living in rural areas, are higher than those of the general adolescent population (14).

The biological immaturity of mothers in pregnancy means "young gynecological age" (before the age of two years from menarche) and growth and development of the mother. In our study, the youngest mother was only 12 years old. Incomplete A case of adolescent multiparty, a 18-yearold quadripara, was also recorded. Underdeveloped cervix bloodstream can lead to subclinical infections, early production of prostaglandins and consequently premature birth (12). This datum, according to our research, shows that leading complications during pregnancy were premature ruptures of membranes, and that leading complications after childbirth were retained placenta and fetal membranes that were 
associated with intrauterine infection (15). Pregnant women who have not completed the physical growth and development are competitors in the developing fetus for nutrients and if we add to that the bad social conditions that they usually come from, malnutrition is mandatory companion of these pregnancies. In addition to these evident medical consequences, pregnancy in adolescence also leads to social isolation, delay or neglect of education, and consequent depression of young mothers (16).

\section{CONCLUSION}

Pregnancy in adolescent period is a major medical, psychological and social problem that society faces. Parent-child communication about sexuality issues is extremely difficult and leads to anxiety and neglecting of the problem, despite the fact that this type of communication as well as sexual education is proven to be associated with postponing sexual intercourse and more consistent use of contraception (17). Firstly, it is necessary to change the attitude of society towards these young people, give them support in the environment they live and provide them with better health care and social treatment. Educating young people about the consequences of risky sexual behavior, as well as presenting them methods of contraception is the best way to prevent serious complications at the beginning of women's generative age.

The necessary steps that society should take are the introduction of education on sexual behavior through elementary education, as well as the involvement of doctors, gynecologists, pediatricians and social workers in the implementation of this education. The role of the gynecologist is to provide the necessary information and to choose adequate contraceptives with the emphasis on the use of long-acting reversible contraceptives (LARC) (18). Improvements in contraceptive use including increases in the use of long-acting reversible contraception and withdrawal in combination with another method-appear to be driving recent declines in adolescent birth and pregnancy rates (19).

Adolescent mothers who initiate a LARC method within 8 weeks of delivery are less likely to have a repeated pregnancy within 2 years than those who choose other methods or no method. First time adolescent mothers should be counseled about the advantage of using LARC (20).

\section{CONFLICT OF INTEREST}

The authors declare that there is no conflict of interest.

\section{REFERENCE}

1. Fraser AM, Brockert JE, Ward RH. Association of Young Maternal Age with Adverse Reproductive Outcomes. The New England Journal of Medicine. 1995; 332(17):1113-1117

2. Demirci O, Yılmaz E, Tosun Ö, Kumru P, Arınkan A, Mahmutoğlu D, Tarhan N. (2016). Effect of Young Maternal Age on Obstetric and Perinatal Outcomes: Results from the Tertiary Center in Turkey. Balkan medical journal, 2016; 33(3):344-349.

3. Brosens I, Benagiano G, Brosens JJ. The potential perinatal origin of placentation disorders in the young primigravida. Am J Obstet Gynecol. 2015;212(5): 580-5.

4. Kang G, Lim JY, Kale AS, Lee LY. Adverse effects of young maternal age on neonatal outcomes. Singapore Med J. 2015;56(3):157-63. doi:10.11622/smedj.20141 94

5. Kaplanoglu M, Bülbül M, Konca C, Kaplanoglu D, Tabak MS, Ata B. Gynecologic age is an important risk factor for obstetric and perinatal outcomes in adolescent pregnancies. Women Birth. 2015;28(4):e119-23.

6. Aitken Z, Hewitt B, Keogh L, LaMontagne AD, Bentley $\mathrm{R}$, Kavanagh AM. Young maternal age at first birth and mental health later in life: Does the association vary by birth cohort? Soc Sci Med. 2016;157:9-17.

7. Treffers PE, Olukoya AA, Ferguson BJ, Liljestrand J. Care for adolescent pregnancy and childbirth. Int J Gynecol Obstet 2001;75:111-121.

8. Varjacic M, Lukic G, Sazdanovic P, Protrka Z, Babic G. Childbirth in adolescence. Gynecology and perinatology 2007;40(3-4):12-4.

9. Varjacic M, Ristic P, Sazdanovic P, Babic G. Adolescent delivery and cesarean section. 7th European congress on Pediatric and Adolescent gynecology, Vienna, Austria .12 - 15 Marth 1997(185).

10. Mijović G, Lukić G, Jokmanović N, Crnogorac S, Kuljić-Kapulica N, Gajić M, Kulauzov M, Bujko M. [Impact of vaginal and cervical colonisation/infection on preterm delivery]. Vojnosanit Pregl. 2008;65 (4):27380.

11. Durutovic Gligorovic S. Anthropometric newborn norms. Medical Faculty of the University of Belgrade. 2000.

12. D Silva AAM, Simeos VMF, Barbieri MA, Betioll H, Lamz Filho F, Coimbra LC and Alves. Young maternal age and preterm birth, Paediatric and Perinatal Epidemiology. 2003;17(4):332-339

13. Part K, Moreau C, Donati S, Gissler M, Fronteira I, Karro H. Teenage pregnancies in the European Union in the context of legislation and youth sexual and reproductive health services. Acta Obstet Gynecol Scand. 2013;92(12):1395-1406.

14. Burrus BB. Decline in Adolescent Pregnancy in the United States: A Success Not Shared by All. Am J Public Health. 2018;108:S5-S6. 
15. Socolov DG, Iorga M, Carauleanu A, Ilea C, Blidaru I, Boiculese L, Socolov RV. Pregnancy during Adolescence and Associated Risks: An 8-Year Hospital-Based Cohort Study (2007-2014) in Romania, the Country with the Highest Rate of Teenage Pregnancy in Europe. Biomed Res Int. 2017;2017:9205016.

16. Leftwich HK, Alves MV. Adolescent Pregnancy. Pediatr Clin North Am. 2017;64(2):381-388.

17. Amie M. Ashcraft, MPHaPamela J.Murray, MHPb Talking to Parents About Adolescent Sexuality. Pediatr Clin North Am. 2017;64(2): 305-320.

18. Powell A. Choosing the Right Oral Contraceptive Pill for Teens. Pediatr. Clin. North. Am. 2017;64:343-358.

19. Laura D. Lindberg, John S. Santelli, Sheila Desai. Changing Patterns of Contraceptive Use and the Decline in Rates of Pregnancy and Birth Among U.S. Adolescents, 2007-2014, Journal of Adolescent Health. 2018;63:253-256.

20. Damle LF, Gohari AC, McEvoy AK, Desale SY, Gomez-Lobo V. Early initiation of postpartum contraception: does it decrease rapid repeat pregnancy in adolescents? J Pediatr Adolesc Gynecol. 2015;28(1):57-62. 